\title{
BIBLIOGRAPHIE
}

[1] A. Tоневотавеv. Biokhimitcheskye osnovy sozrevanya syrov, Moscou, 1959.

[2] E. Pijanowski. Zarys chemii i technologii mleczarstwa. Varsovie, 1956.

[3] J. Kurmann. Dtsche Molkerei Ztg., 78, 1146, 1957.

[4] H. F $\triangle$ Gen, J. Stine et R. Hussong. J. Dairy Sci, 35, 779, 1952.

[5] G. PUlay. XIVe Congrès Int. Laiterie, 2, 432, 1956.

[6] G. SృöströM. XIVe Congrès Int. Laiterie, 3, 562, 1956.

[7] R. Nilson et M. Guldstrand. XVe Congrès Int. Laiterie, 3, 1773, 1959.

[8] P. Swarting et S. Matisson. XiII Congrès Int. Laiterie, 2, 615, 1953.

[9] C. W. RaAdsveld. Versl. Ned. Inst. Zuivelonderzoek, no 27, 1957.

[10] T. Nakanishi et F. Tokita. J. Agr. Res., 9, 37, 1958.

[11] S. Tuckey et M. SaHasrabudha. J. Dairy Sci., 39, 918, 1956.

[12] E. Lipinska. Prace Inst. Przem. Mlecz. Varsovie, 8, 1, 1961.

[13] P. TAYlor et L. Clegg. J. Dairy Res., 25, 32, 1958.

Cette étude fait partie du travail exécuté avec les fonds de United States Department of Agriculture, Agricultural Research Service. (FG-PO-151). Les auteurs expriment leur vive reconnaissance à cet Organisme.

\section{MESURE DE LA TENSION SUPERFICIELLE DE QUELQUES LAITS \\ AU TENSIOMĖTRE A ANNEAU \\ DE LECOMTE DU NOUY (1)}

par

\section{A. CALANDRON}

Ecole Nationale Supérieure

Agronomique de Rennes.

\section{GRILLET}

Faculté des Sciences

de Rennes

\section{Remarques préliminaires}

Afin d'éviter les erreurs de mesure de la tension superficielle dues soit aux variations de la température du liquide à mesurer, soit aux vapeurs ou gaz de l'atmosphère, nos essais furent effectués dans un grand laboratoire de physique ne servant, au cours de la journée, qu'aux expériences décrites et seulement occupé par les auteurs de cette Note.

(1) Bull. Soc. sc. Bretagne. (Bulletin 37, paru en avril 1964.) 
En général, les laits étaient amenés à la température suffisamment constante du laboratoire, soit 22,5 à $23^{\circ} \mathrm{C}$. Lorsque la température d'essai fut différente, nous l'avons précisée.

La mesure du lait, est délicate. Après différents essais, nous estimons bon de placer l'anneau dans une position telle qu'il se sépare de lui-même après une attente de cinq à dix secondes. L'expérience recommencée plusieurs fois donne des résultats consécutifs identiques, aux erreurs d'expérience près. Dans les conditions adoptées, les écarts dans les résultats atteignent au maximum plus ou moins 0,2 dyne $/ \mathrm{cm}$.

Le lait est bien mélangé par transvasements en évitant la mousse; la mesure est effectuée aussitôt. Si la période d'attente est d'une heure et demi à deux heures, la couche de crème est visible et la tension diminue de 2,5 à $3 \mathrm{dyn} / \mathrm{cm}$ par rapport au chiffre du lait non reposé. Le nombre de transvasement ne paraît pas avoir d'influence, mais à condition d'éviter la mousse, conséquence d'une forte agitation inutile.

\section{Résultats de quelques essais}

1) Lait de vache $\mathrm{E} .$. de race hollandaise.

Lait de la traite du soir. Richesse du lait: $34,5 \mathrm{~g}$ de matière grasse et $121 \mathrm{~g}$ d'extrait sec par litre. Acidité : 16,5 degrés Dornic. $48 \mathrm{dyn} / \mathrm{cm}$ à $20^{\circ} \mathrm{C}$ et $46,5 \mathrm{dyn} / \mathrm{cm}$ à $23^{\circ} \mathrm{C}$.

2) Lait de la vache D... de race hollandaise.

Lait de la traite du soir. Richesse du lait: 37,5 de matière grasse et $125,8 \mathrm{~g}$ de matières sèches par litre. Acidité : 16 degrés Dornic.

47,5 à $48 \mathrm{dyn} / \mathrm{cm}$ à $20^{\circ} \mathrm{C}$. Après 45 minutes de repos dans le petit cristallisoir à essais : 46,5 à $47 \mathrm{dyn} / \mathrm{cm}$.

Laps de temps entre le moment de la traite et le moment de la mesure : environ une heure.

3) Lait de la traite du soir de la Vache Dora II.

Vache traite le 5 juillet 1962.

Richesse du lait : $38,5 \mathrm{~g}$ de matière grasse ; $124,8 \mathrm{~g}$ de matières sèches par litre,.

Le lait est gardé à l'armoire frigorifique dès la traite $\left(+1^{0}\right.$ à $+2^{\circ} \mathrm{C}$ ) jusqu'au lendemain dix heures du matin. Il est dilué avec de l'eau fraîchement distillée dans du verre dans les proportions de $5,10,20,40,60,80,90$ et 95 parties pour $100 \mathrm{du}$ mélange.

Les mesures de tension ont été faites cinq heures après le moment des dilutions. Les dilutions sont gardées au frais, 18 à $20^{\circ} \mathrm{C}$ 
environ 3 heures et elles sont amenées à la température de la pièce d'essais deux heures avant la mesure.

La température du laboratoire est de $22,5^{\circ} \mathrm{C}$, celle des laits est la même.

Le tableau suivant donne les résultats de ces essais :

Volumes d'eau pour

100 volumes du mélange $\quad \begin{array}{lllllllll}0 & 5 & 10 & 20 & 40 & 60 & 80 & 90 & 95\end{array}$

Dyn/em

$\begin{array}{lllllllll}47,5 & 46,5 & 43,0 & 41,0 & 41,0 & 43,5 & 46,5 & 47,2 & 47,5\end{array}$

Nous signalons l'absence de résultats pour les mouillages compris entre 20 et 40 parties d'eau pour 100 du mélange $(\nabla / v)$.

4) Lait de ramassage d'une usine rennaise et lait écrémé recueilli à la sortie de l'écrémeuse.

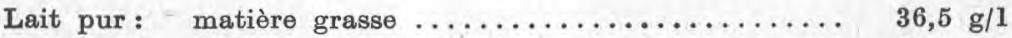

matières sèches.................. 121,5

tension superficielle à $22^{\circ} \mathrm{C} \ldots \ldots \ldots \ldots \ldots \ldots, 48 \mathrm{dyn} / \mathrm{cm}$

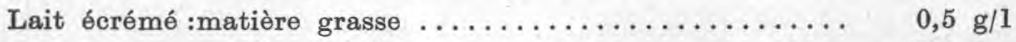

matières sèches.................. 90,1

tension superficielle à $22^{\circ} \mathrm{C} \ldots \ldots \ldots \ldots \ldots \ldots \quad 46 \quad \mathrm{dyn} / \mathrm{em}$

L'acidité de ces laits est de 17 à $18^{\circ}$ Dornic, soit la même acidité qu'une solution aqueuse d'acide lactique à 1,7-1,8 g par litre.

Le lait sortant de l'écrémeuse était chaud: $43-45^{\circ} \mathrm{C}$. Il fut gardé trois heures à $22^{\circ} \mathrm{C}$ avant les mesures.

$\mathrm{Au}$ moment des mesures, chaque échantillon est bien mélangé par transvasements.

Le lait pur fut prélevé dans le grand bac de garde de l'usine. Avec le type d'écrémeuse industrielle, il n'était pas possible de faire un prélèvement immédiatement avant l'entrée du lait dans la centrifugeuse. Il s'ensuit que les deux laits, pur et écrémé, ne sont pas comparables, ce qui est confirmé par les différences d'extrait sec dégraissé : lait pur 85 et lait écrémé $89,6 \mathrm{~g} / 1$.

5) Lait concentré non sucré.

Lait en boîte métallique, marque Gloria.

Indications de l'industriel : en ajoutant au contenu de la boîte $435 \mathrm{~g}$ d'eau bouillie, on obtient 82 centilitres de lait à $35 \mathrm{~g}$ de matière grasse par litre. Matière grasse du lait concentré : 7 p. 100 . Lait homogénéisé et concentré sous vide à basse température.

a) Température de mesure : $22^{\circ} \mathrm{C}$.

Tension superficielle du lait concentré : 52 à $53 \mathrm{dyn} / \mathrm{cm}$. 
b) Température de mesure : $22^{\circ} \mathrm{C}$.

Tension superficielle du lait concentré mouillé en progression croissante avec de l'eau fraîchement distillée :

Pour un volume de lait concen-

$\begin{array}{ccccccccc}\text { tré, volumes d'eau ajoutée } & 0 & 1 & 3 & 7 & 15 & 31 & 63 & 127 \\ \text { dyn/cm } & 52-53 & 52 & 51-52 & 49 & 49 & 49 & 49,5-50 & 49,5-50\end{array}$

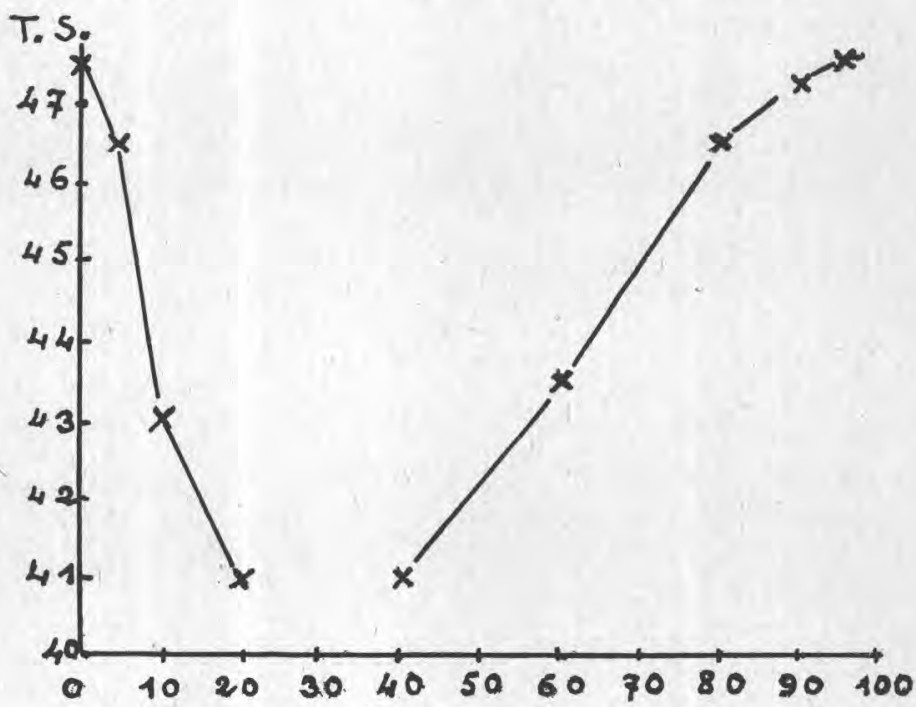

Diagramme de la tension superficielle du lait de la vache Dora II.

- En abscisse : volumes d'eau pour 100 volumes du mélange.

- En ordonnée: T.S. en dynes par cm.

6) Mélange à parties égales du lait de la vache Dora II mouillé à 40 volumes d'eau pour 100 volumes de mélange, avec le lait concentré non sucré Gloria mouillé à raison de 1 partie de lait pour 127 parties d'eau (en volume).

Le mélange de ces deux laits mouillés et dont les tensions superficielles sont respectivement de $41 \mathrm{dyn} / \mathrm{cm}$ pour le lait de vache mouillé, et de près de $50 \mathrm{dyn} / \mathrm{cm}$ pour le lait concentré mouillé, donne une tension superficielle de $41 \mathrm{dyn} / \mathrm{cm}$. Cette expérience confirme le fait bien connu que la valeur de la tension superficielle est liée à la structure de la couche superficielle.

7) Le lait de vache Dora II, additionné de $1 \mathrm{~g}$ par litre de bichromate de potassium, gardé trois jours vers $+2^{\circ} \mathrm{C}$, bide mélangé, pur, ramené à $22^{\circ} \mathrm{C}$, donne une tension superficielle en $46 \mathrm{dyn} / \mathrm{cm}$. 


\title{
Conclusion
}

Des laits individuels de vaches de race hollandaise de la région de Rennes, ayant des teneurs en matière grasse de 34,5 à $38 \mathrm{~g}$ par litre, ont une tension superficielle de 47,5 à $48 \mathrm{dyn} / \mathrm{cm}$ au tensiomètre de Lecomte du Nouy pour des mesures à 20-220 C. Dans les conditions adoptées, les résultats peuvent varier de plus ou moins $0,2 \mathrm{dyn} / \mathrm{cm}$ autour de la moyenne.

Le mouillage abaisse la tension superficielle jusqu'à un minimum d'environ $41 \mathrm{dyn} / \mathrm{cm}$ pour des mouillages de 20 à 40 volumes d'eau pour 100 volumes de mélange. Au-delà de 40 p. 100, la tension se relève. Pour de très fortes dilutions aqueuses, la tension se rapproche de la tension du lait pur sans l'atteindre.

Avec des laits récemment traits, ou correctement gardés quelques jours, en tout cas non altérés, un faible mouillage abaissant la tension superficielle d'une façon appréciable, cette mesure pourraît être prise en considération pour confirmer les analyses d'un lait suspect de fraude par mouillage.

La tension superficielle d'un lait concentré non sucré, homogénéisé, ayant 7 p. 100 de matière grasse, est de 52 à $53 \mathrm{dyn} / \mathrm{cm}$ à $22,5^{\circ}$ C. Le mouillage diminue la tension superficielle jusqu'à $49 \mathrm{dyn} / \mathrm{cm}$. Elle se relève légèrement pour de très fortes dilutions, sans dépasser $50 \mathrm{dyn} / \mathrm{cm}$.

\section{L'UTILISATION DE COLORANTS \\ POUR déceler LA PRÉsence d'ANTIBIOTIQUES DANS LE LAIT (1)}

\author{
par \\ P. KASTLI
}

\section{I. - Introduction}

Les rapports établis précédemment par le Pr STORGaRDs pour la commission F.I.L. de la Production du Lait et publiés dans le Bulletin de la Fédération en 1962 (partie III), traitent de façon complète et détaillée des procédés mis en œuvre pour déceler la présence d'antibiotiques dans le lait. On dispose actuellement de

(1) Bull. F.I.L., 1963, VI (Rapport présenté par M. le Professeur Docteur P. Kästli et reproduit avec l'autorisation de la Fédération Internationale de Laiterie). 